\title{
16. THE LINE SPECTRA OF WHITE DWARFS
}

\author{
P. A. STRITTMATTER and D. T. WICKRAMASINGHE \\ Institute of Theoretical Astronomy, Cambridge, U.K.
}

\begin{abstract}
Model atmospheres with temperatures in the range $10000 \leq T_{\text {eff }} \leq 25000 \mathrm{~K}$ and with gravities $6 \leq \log (g) \leq 9$ have been constructed for various helium abundances with a view to understanding the spectra of the hotter white dwarfs. It is shown that the DB stars are confined to the temperature range $15000 \leq T_{\text {eff }} \leq 18000 \mathrm{~K}$ in which convection also becomes important in the outer layers of helium rich stars. The DA stars seem to avoid this temperature range. The hydrogen and metal abundances in DB atmospheres are shown to be reduced by factors $10^{5}$ and $10^{3}$ respectively compared to solar values. Possible explanations of DB and DC spectra are discussed.
\end{abstract}

\section{Introduction}

A considerable amount of observational data on the photometric and spectroscopic properties of white dwarfs has now been assembled, largely by Eggen and Greenstein $(1965,1967)$, Eggen $(1970)$, Greenstein $(1960,1969)$. On the other hand comparatively little progress has so far been made in the theoretical interpretation of this data, mainly perhaps due to the lack of an adequate description of atomic processes in high density plasmas. While certain theoretical difficulties still exist, particularly in computing opacities and ionisation equilibria at high densities, enough is now known to allow calculation of reasonably accurate model atmospheres for these stars. Such models have accordingly been computed by us for a range of effective temperatures, gravities and compositions. In this paper we shall use the computed line strengths and photometric data to analyse the gross features of the spectra of hot $\left(T_{\mathrm{eff}} \gtrsim 10000 \mathrm{~K}\right)$ white dwarfs. A detailed account of this work is given in Paper III (Strittmatter and Wickramasinghe, 1970).

\section{The Model Atmosphere and Line Profile Calculations}

For each set of input parameters (effective temperature, gravity and composition) a constant flux model atmosphere in radiative equilibrium has been computed using the basic procedure outlined by Avrett and Krook (1963). Where Lyman and Balmer line blanketing is important it has been taken into account using a modified temperature correction procedure (Wickramasinghe and Strittmatter, 1969). The effect of blanketing by $\mathrm{HeI}$ lines on the structure of the atmosphere is negligible at the temperatures considered here because the peak continuum flux never coincides with the position of strong lines. In each case flux constancy was achieved to an accuracy of better than $1 \%$. Some of the atmospheres turned out to be convectively unstable in certain optical depth ranges; the effect has not been taken into account in our present calculations since, to the accuracy required here, the resultant changes in the observable parameters have been shown to be small (Wickramasinghe and Strittmatter, 1970; Paper II). 
The sources of continuum opacity included are $\mathrm{H}, \mathrm{H}^{-}, \mathrm{H}_{2}, \mathrm{He}, \mathrm{He}^{+}, \mathrm{He}^{-}$and electron scattering. The $\mathrm{He}^{-}$absorption coefficient has been taken from Somerville (1965); the remainder are treated as specified by Vardya (1961). Absorption from the bound states of metals has not been taken into account although it could cause substantial blanketing at far ultra-violet wavelengths particularly in the hydrogen deficient stars. This omission will generally cause our models to appear slightly cooler than would otherwise be the case, but has been made (a) for computational economy and (b) because it appears from the observations that the metal abundance in the surface layers of most white dwarfs is very low.

The HeI $\lambda 4472$ line has been computed using the detailed profiles of Griem (1968). The forbidden component at $\lambda 4517$ is included in the computation of the equivalent width and may result in over-estimation of this quantity as compared to the observations (Paper II). We have also computed the lines $\mathrm{HeI} \lambda 4713, \mathrm{He}$ I $\lambda 5875, \mathrm{Mg}$ II $\lambda 4481$, SiII $\lambda 4129$, CaII $(H+K), C_{I I} \lambda 4267$ and FeI $\lambda 4272$. The broadening mechanisms included in those calculations are van der Waal's broadening by $\mathrm{He}$ and $\mathrm{H}$, quadratic Stark broadening, Doppler broadening and radiation damping; the latter two are generally negligible in white dwarf atmospheres.

The UBV photometric parameters have been computed from the theoretical energy distribution (continuum and hydrogen lines) using the prescription of Matthews and Sandage (1963).

The basic models may be divided into two groups. The first group consists of a

\section{TABLE I}

Photometry and equivalent widths for several models

\begin{tabular}{|c|c|c|c|c|c|c|c|c|}
\hline$T_{\text {eff }} / 10^{3}$ & $\log (g)$ & $N_{\mathrm{He}}$ & $B-V$ & $U-B$ & B.C. & $T_{\text {eff }} \mathrm{W}\left(\mathrm{H}_{\gamma}\right)$ & $\mathrm{W}\left(\mathrm{He}_{\mathrm{I}} \lambda 4472\right)$ & $V$ \\
\hline 10 & 8 & 0.144 & 0.21 & -0.66 & -0.56 & 30.2 & 0 & 12.86 \\
\hline 12 & 8 & 0.144 & 0.19 & -0.62 & -0.70 & 49.9 & 0 & 12.21 \\
\hline 15 & 8 & 0.144 & 0.07 & -0.74 & -1.12 & 48.5 & 0 & 11.66 \\
\hline 20 & 8 & 0.144 & -0.07 & -0.94 & -1.88 & 36.0 & 2.19 & 11.17 \\
\hline 25 & 8 & 0.144 & -0.17 & -1.04 & -2.47 & 28.0 & 6.27 & 10.79 \\
\hline 10 & 8 & 100.0 & 0.06 & -0.88 & -0.79 & 11.6 & 0 & 13.09 \\
\hline 15 & 8 & 100.0 & 0.02 & -0.90 & -1.25 & 36.4 & 4.94 & 11.79 \\
\hline 20 & 8 & 100.0 & -0.16 & -1.04 & -1.82 & 17.9 & 22.4 & 11.11 \\
\hline 25 & 8 & 100.0 & -0.27 & -1.10 & -2.40 & 0.1 & 22.3 & 10.72 \\
\hline 10 & 8 & 1000.0 & 0.01 & -0.92 & -0.88 & 3.45 & 0.0 & 13.17 \\
\hline 15 & 8 & 1000.0 & -0.10 & -1.04 & -1.43 & 11.5 & 5.18 & 11.97 \\
\hline 20 & 8 & 1000.0 & -0.23 & -1.04 & -1.79 & 1.58 & 29.4 & 11.08 \\
\hline 25 & 8 & 1000.0 & -0.27 & -1.08 & -2.51 & 0 & 24.4 & 10.83 \\
\hline 10 & 8 & 10000.0 & 0.01 & -0.92 & -0.87 & 0 & 0 & 13.17 \\
\hline \multirow[t]{2}{*}{15} & 8 & 10000.0 & -0.15 & -1.07 & -1.53 & 1.06 & 5.99 & 12.07 \\
\hline & 7 & & -0.15 & -1.05 & -1.47 & 3.78 & 8.86 & 12.01 \\
\hline \multirow[t]{2}{*}{20} & 8 & 10000.0 & -0.23 & -1.03 & -1.88 & 0 & 26.2 & 11.17 \\
\hline & 7 & & -0.23 & -1.01 & -1.88 & 0 & 23.8 & 11.18 \\
\hline \multirow[t]{2}{*}{25} & 8 & 10000.0 & -0.27 & -1.07 & -2.58 & 0 & 29.2 & 10.9 \\
\hline & 7 & 10000.0 & -0.27 & -1.06 & -2.58 & 0 & 22.7 & 10.9 \\
\hline
\end{tabular}


grid of twenty model atmospheres with $\log (g)=6,7,8,9$ and $T_{\text {eff }}=10000,12000$, $15000,20000,25000 \mathrm{~K}$ and are of 'normal' composition $\left(N_{\mathrm{He}}=0.144, n_{m} / n_{\mathrm{H}}=0.00165\right)$. The second group of fifteen model atmospheres has input parameters $\log (g)=8$, $T_{\text {eff }}=10000,15000,20000,25000 \mathrm{~K}, N_{\mathrm{He}}=10^{2}, 10^{3}, 10^{4} ;$ and $\log (g)=7, T_{\text {eff }}=15000$, $20000,25000, N_{\mathrm{He}}=10^{4}$. The metal abundance in the second, helium rich group was fixed by taking $N_{m}=n_{m} / n_{\mathrm{He}}=10^{2}$. A second computation of certain line strengths was carried out for the second group using metal abundances reduced by a factor 10 but the atmospheric structure was assumed to be the same as before.

In Table I we list photometry and equivalent widths of $\mathrm{H} \gamma$ and $\mathrm{HeI} \lambda 4472$ for a range of models with increasing helium content. The effects of the HeI lines on the UBV colours has not been included in these calculations (see discussion below).

In Figure 1 we have plotted equivalent widths of $\mathrm{H} \gamma$ against $\mathrm{U}-\mathrm{V}$ for a range of models with $\log g=8$ but different helium abundances. For the normal DA stars equivalent widths of up to $50 \AA$ occur, falling however, very sharply once $\mathrm{H}^{-}$opacity sets in at $12000 \mathrm{~K}$.

The HeI $\lambda 4472$ equivalent width is plotted against $U-V$ in Figure 2. Values of W(4472) of nearly $30 \AA$ are reached for the high helium abundances. The sharp drop-off in equivalent width for $T \lesssim 20000 \mathrm{~K}$ in these models is due to $\mathrm{He}^{-}$opacity. The influence of the latter source is maintained until $T=25000 \mathrm{~K}$ in the case when $\log g=8$ and $N_{\mathrm{He}}=10^{4}$, thus accounting for the continuing rise with temperature of $\mathrm{W}(4472)$. For $\log g=7$, however, the $\mathrm{He}^{-}$ceases to be of importance for $T \sim 20000 \mathrm{~K}$

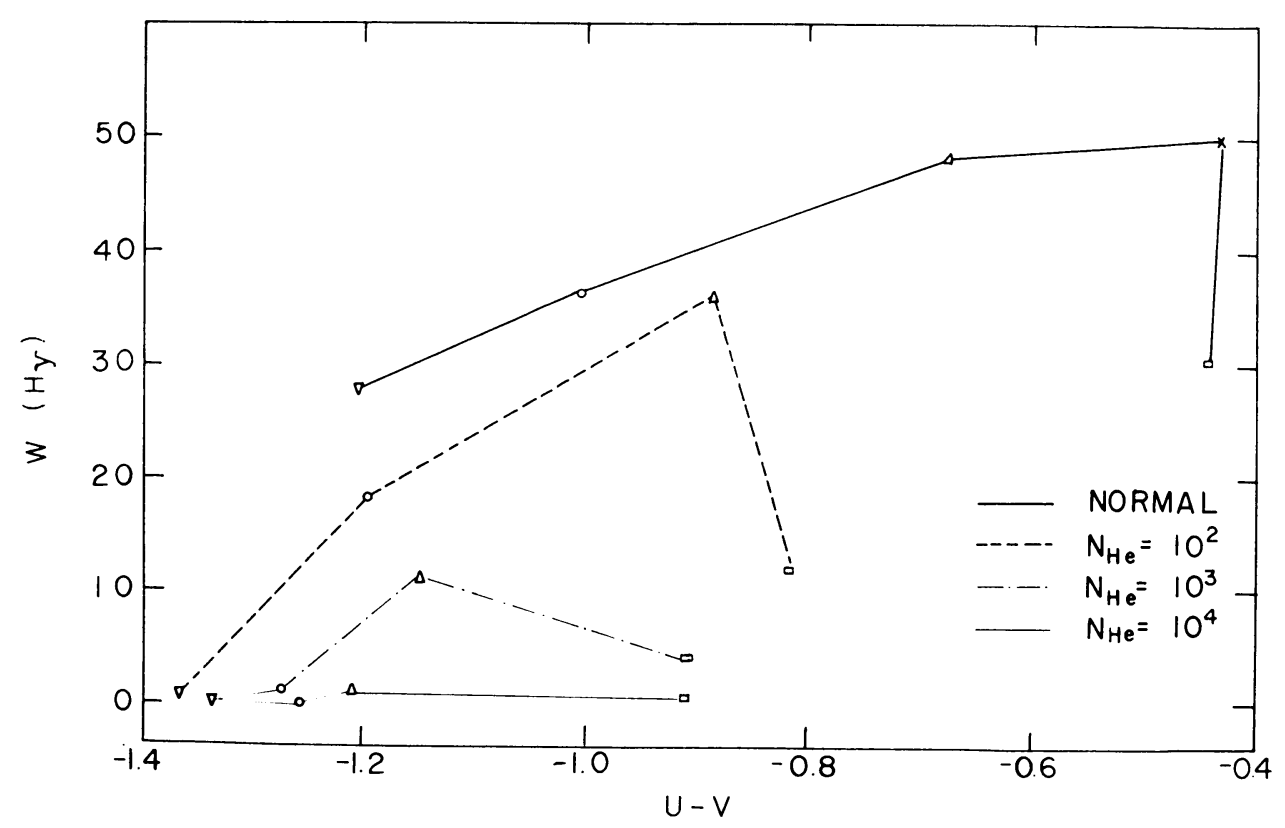

Fig. 1. A plot of the equivalent width of $H \gamma$ against $(\mathrm{U}-\mathrm{V})$ for the various models with $\log (g)=8$. The squares, triangles, circles and inverted triangles correspond to models with $T_{e}=10000 ; 15000$; $20000 ; 25000 \mathrm{~K}$ respectively. The symbol $X$ is used to denote a $T_{e}=12000 \mathrm{~K}$ model. 


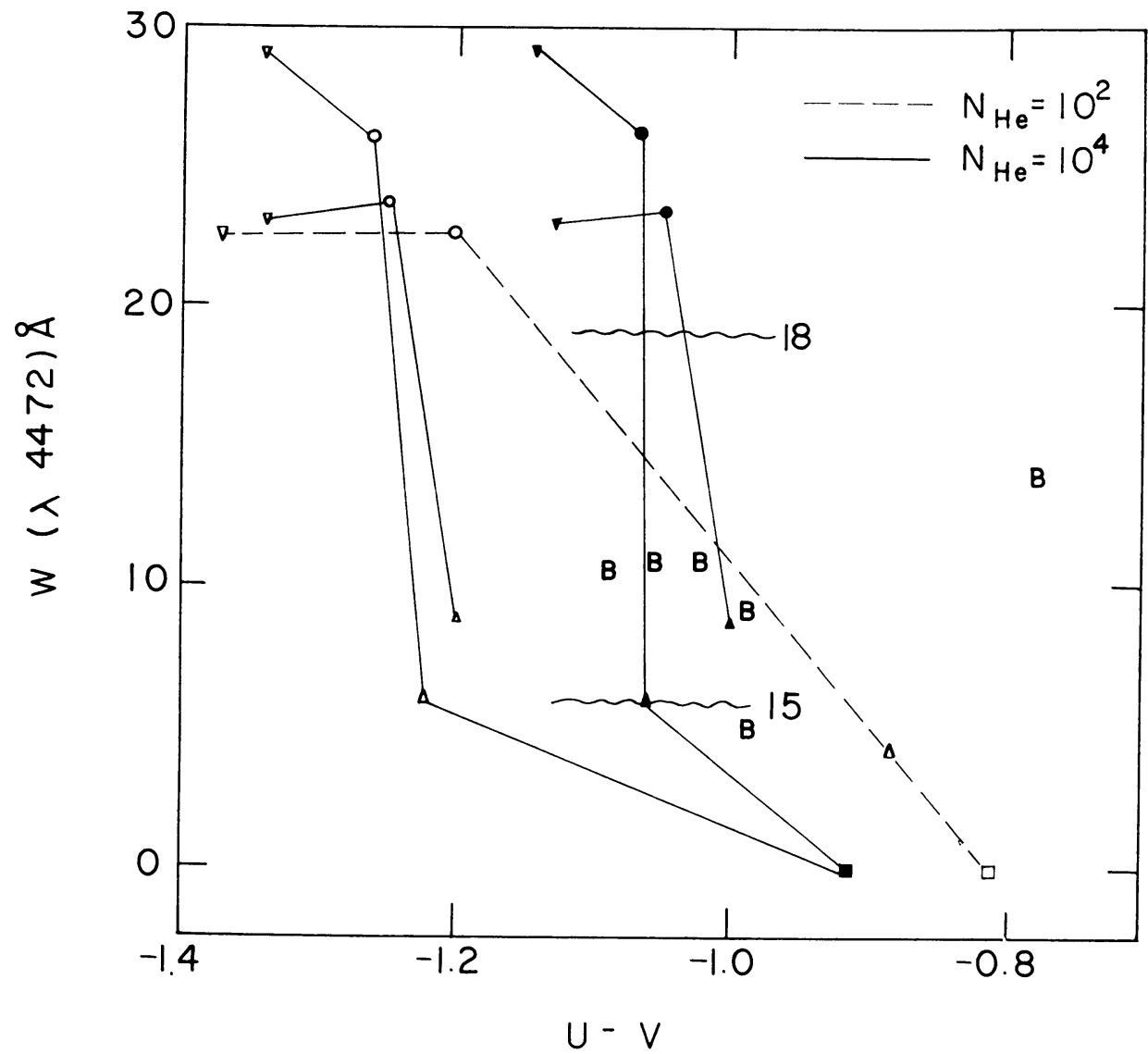

Fig. 2. A plot of the equivalent width of HeI ( 24472$)$ against (U - V) for the helium rich models. Smaller symbols denote models with $\log (g)=7$. All other models have $\log (g)=8$. Open symbols denote colours computed without inclusion of the HeI lines, solid symbols those in which the lines have been taken into account by the approximate method (see text). The letter B is used to denote the positions of the observed DBs with known W( $\lambda 4472)$. Otherwise the notation is as in Figure 1.

and $\mathrm{W}(4472)$ accordingly decreases with further increases in temperature owing to the decrease in the Boltzmann ratio of level populations giving rise to the HeI line and continuum opacity respectively. From Table I we note that for intermediate compositions $\left(1 \lesssim N_{\mathrm{He}} \lesssim 10^{3}\right)$ both the $\mathrm{H}$ and $\mathrm{HeI}$ lines are very strong. We may conclude from the fact that no such stars have yet been observed that this range of composition is very rare. Only when $N_{\mathrm{He}} \gtrsim 10^{4}$ are the hydrogen lines negligible over the entire temperature range. White dwarf atmospheres are either hydrogen rich or extremely hydrogen deficient.

At this point, however, we should consider the effect of $\mathrm{He}_{\mathrm{I}}$ lines on the computed colours. It is quite clear from Figure 2 that W(4472) would be most easily observable for $N_{\mathrm{He}}=10^{4}$ at colours much bluer $(\mathrm{U}-\mathrm{V}=-1.2)$ than those observed 
$(\mathrm{U}-\mathrm{V}=-1.0)$. However, both the $\mathrm{U}$ and $\mathrm{B}$ bands contain large numbers of lines and these will clearly have the effect of reddening $\mathrm{U}-\mathrm{V}$ since only two He lines, $\lambda 5875$ and $\lambda 6678$ appear in the $\mathrm{V}$ band. The task, however, of computing each line individually in order to calculate broad band photometric parameters is not only immense but extremely misguided. Alternative observational parameters will therefore be given in Paper IV. Since much of our present knowledge is in the form of UBV data, however, we shall give an approximate method for estimating the blanketing effects.

Klemola (1961) has given line strengths of more than fifty HeI lines in the spectrum of the helium star $\mathrm{BD}+10^{\circ} 2179$. We have accordingly taken these values, bunched them in $50 \AA$ intervals and scaled them according to the ratio of our computed value of $\mathrm{W}(4472)$ to that observed in $\mathrm{BD}+10^{\circ} 2179$. These 'pseudo lines' were then included in the fluxes from which UBV photometry was computed. The fact that for more closely spaced lines the full effect of growth in equivalent width will not be obtained was taken into account in an approximate way. The resultant corrections to the colours of the various models are shown in Table II. We do not claim that they are

TABLE II

Corrections to colours for various models

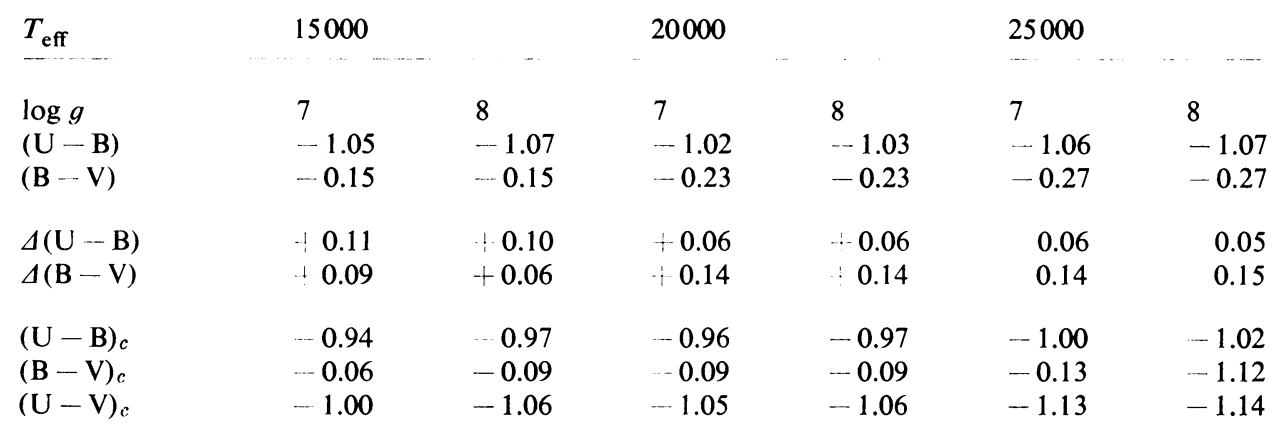

accurate, merely that they give a reasonable estimate for the effect. While the corrections to $\mathrm{U}-\mathrm{V}$ and $\mathrm{B}-\mathrm{V}$ are substantial at high values of $\mathrm{W}(4472)$, the change in $U-B$ is small. This results from the higher density of lines in the $U$ band which accordingly reaches saturation at somewhat lower values of W(4472) than does the $B$ band. Thus while the initial $U-B$ change is strongly redwards it then reverses with increasing W(4472) until both $U$ and B bands are effectively 'saturated'. We note that the computed corrections cause a substantial reddening in $U-V$ for $T \gtrsim 15000 \mathrm{~K}$ causing the drop in W(4472) with colour to be even steeper than shown in Figure 2. It is also clear from Figure 2 that both the colours and equivalent widths of $\mathrm{He}$ I $\lambda 4472$ agree well with the observations for $15000 \lesssim T \lesssim 18000 \mathrm{~K}$.

Finally we list in Table III the strengths of the following additional lines; He I $\lambda 4713$, HeI $\lambda$ 5875, MgII $\lambda 4481$, SiII $\lambda$ 4129, CaII $(H+K), C_{I I} \lambda 4267$ and FeI $\lambda 4272$. The 
TABLE III

Strengths of additional lines

\begin{tabular}{|c|c|c|c|c|c|c|c|c|c|}
\hline $\begin{array}{l}T_{\text {eff }} \\
10^{3}\end{array}$ & $\log g$ & $N_{\mathrm{He}}$ & $\frac{\mathrm{He} \mathrm{I}}{\lambda 5875}$ & $\frac{\mathrm{HeI}}{\lambda 4713}$ & $\begin{array}{l}\mathrm{Mg}_{\mathrm{II}} \\
\lambda 4481\end{array}$ & $\begin{array}{c}\text { Si II } \\
\lambda 4129\end{array}$ & $\begin{array}{c}\text { Ca II } \\
(\mathrm{H}+\mathrm{K})\end{array}$ & $\begin{array}{c}\text { CII } \\
\lambda 4267\end{array}$ & $\frac{\mathrm{FeI}_{\mathrm{I}}}{\lambda 4272}$ \\
\hline & & & \multicolumn{7}{|c|}{ Normal metal abundance } \\
\hline 10 & 8 & 0.144 & +0 & 0 & 0.449 & 0.099 & 2.35 & 0 & 0.210 \\
\hline 15 & 8 & 0.144 & 0.326 & 0.028 & 0.734 & 0.314 & 0.138 & 0.012 & 0 \\
\hline 20 & 8 & 0.144 & 1.77 & 0.381 & 0.5 & 0.391 & 0.046 & 0.106 & 0 \\
\hline 25 & 8 & 0.144 & 4.433 & 1.21 & 0.3 & 0.274 & 0.016 & 0.336 & 0 \\
\hline
\end{tabular}

High metal abundance $\left(N_{m}=10^{-2}\right)$

$\begin{array}{cccccccccc}10 & 8 & 10000.0 & 0.028 & 0 & 5.63 & 1.6 & 80.4 & 0 & 16.3 \\ 15 & 8 & 10000.0 & 3.76 & 0.784 & 16.5 & 5.90 & 29.9 & 0.255 & 2.53 \\ & 7 & & 4.29 & 1.68 & 15.64 & 5.90 & 16.5 & 0.462 & 0.783 \\ 20 & 8 & 10000.0 & 22.6 & 8.27 & 8.34 & 4.87 & 0.832 & 1.45 & 0.025 \\ & 7 & & 14.8 & 5.68 & 3.82 & 2.88 & 0.249 & 1.04 & 0.008 \\ 25 & 8 & 10000.0 & 23.5 & 8.69 & 3.10 & 2.41 & 0.145 & 1.44 & 0 \\ & 7 & & 13.5 & 5.03 & 1.22 & 1.16 & 0.070 & 0.988 & 0\end{array}$

Low metal abundance $\left(N_{m}=10^{-3}\right)$

$\begin{array}{llllllllll}10 & 8 & 10000.0 & 0.028 & 0 & 1.55 & 0.307 & 28.9 & 0 & 5.22 \\ 15 & 8 & 10000.0 & 3.76 & 0.784 & 5.15 & 1.91 & 9.77 & 0.032 & 0.942 \\ & 7 & & 4.29 & 1.68 & 4.94 & 2.11 & 5.27 & 0.104 & 0.283 \\ 20 & 8 & 10000.0 & 22.6 & 8.27 & 2.58 & 1.72 & 0.277 & 0.371 & 0.004 \\ & 7 & & 14.8 & 5.68 & 1.20 & 1.04 & 0.085 & 0.334 & 0.0 \\ 25 & 8 & 10000.0 & 23.5 & 8.69 & 0.905 & 0.769 & 0.046 & 0.374 & 0.0 \\ & 7 & & 13.5 & 5.03 & 0.391 & 0.369 & 0.026 & 0.322 & 0.0\end{array}$

results are for $\log g=8$ and $N_{\mathrm{He}}=0.144$ and $10^{4}$ respectively, it having been already demonstrated that intermediate cases are rare if they exist at all. For the high helium abundance, results for two values of $N_{m}=10^{-2}$ and $10^{-3}$ and for $\log g=7$ are given.

\section{Conclusions}

It has been noted by Greenstein (1969) that the very existence of DB stars is remarkable if accretion of interstellar matter can occur. For a typical DB white dwarf, moving through the interstellar medium with a velocity of $30 \mathrm{~km} / \mathrm{sec}$, and accreting matter at the Eddington rate, the time taken to accrete 'one' atmosphere is $\sim 10^{3} \mathrm{yr}$. If a tail shock can form and destroy momentum perpendicular to the direction of motion of the star, this accretion time scale is reduced to $\sim 10^{-1} \mathrm{yr}$ (Bondi and Hoyle, 1944). As we have shown in the previous section, only a small amount of hydrogen is sufficient to produce detectable hydrogen lines. We have accordingly investigated various mechanisms for preventing accretion. The roles of convection, circulation and gravi- 
tational diffusion are also examined (Paper III). Our main conclusions may be summarised as follows.

(i) The hydrogen abundance in DB white dwarfs must be at least $10^{5}$ times smaller relative to helium than in normal stars.

(ii) The helium abundance in DA white dwarfs cannot exceed the normal value.

(iii) No intermediate hydrogen to helium ratios have yet been observed.

(iv) Metal abundances in DB stars must be at least a factor $10^{3}$ lower than the solar value.

(v) Most DB stars lie in the temperature range $15000 \lesssim T_{\text {eff }} \lesssim 18000 \mathrm{~K}$ at which convection becomes important in the outer layers.

(vi) DA stars apparently avoid the temperature range occupied by the DB stars.

(vii) Accretion should be important except in high velocity white dwarfs with moderately strong magnetic fields. It should occur in the DB stars.

(viii) Gravitational diffusion of heavy elements could be of importance in the presence of a magnetic field. In general a field strong enough to prevent accretion will allow diffusion to occur.

We suggest that these findings may be understood (hypothesis A) if most white dwarfs have helium rich envelopes and fairly weak magnetic fields. They therefore accrete interstellar material which resides on the surface and allows them to cool as normal DA stars. When the helium recombines convection is set up which removes the accreted material and mixes it downward. This surface convection has been shown to occur in the temperature range $15000 \lesssim T_{\text {eff }} \lesssim 18000 \mathrm{~K}$. The difficulties with this hypothesis, in particular the problems of stability of the cooler atmospheres and dilution of accreted material, lead to some doubts despite the satisfactory observational picture.

As an alternative we propose that accretion can be prevented in DB stars perhaps due to rotation coupled to a moderately strong magnetic field (hypothesis B). The concentration of stars in the range $15000 \lesssim T_{\text {eff }} \lesssim 18000 \mathrm{~K}$ could then be attributed to the establishment of a deep convective zone in the envelopes of these stars (van Horn, 1970). The apparent absence of DA stars in this temperature range would, however, have to be attributed to chance in a small sample.

We also propose that there is a class of 'peculiar' white dwarfs, with moderately high space motions and above average magnetic fields. Accretion is thus prevented and diffusion of heavy elements downward can occur. These stars accordingly cool like DBs until the temperature falls below $\sim 12000 \mathrm{~K}$ when the HeI lines disappear. Provided metal diffusion is efficient enough the star would then appear as a hot $\mathrm{DC}-\mathrm{He}^{-}$providing the opacity source. As on the main sequence 'peculiar' spectra would thus be associated with the presence of a magnetic field. On hypothesis B all DB stars could give rise to DC spectra at lower temperatures.

It appears to us that hypothesis $A$ is perhaps in more obvious agreement with the observational evidence, but that hypothesis $\mathrm{B}$ raises fewer difficulties of principle (particularly since it does not demand large numbers of hydrogen and metal deficient, low velocity white dwarfs). 


\section{References}

Avrett, F. H. and Krook, M.: 1963, Astrophys. J. 137, 874.

Bondi, H. and Hoyle, F.: 1944, Monthly Notices Roy. Astron. Soc. 104, 273.

Brechot-Sahal, S.: 1968, Z. Astrophys. 69, 74.

Eggen, O. J.: 1970, Astrophys. J. 159, 945.

Eggen, O. J. and Greenstein, J. L.: 1965, Astrophys. J. 141, 83.

Eggen, O. J. and Greenstein, J. L.: 1967, Astrophys. J. 150, 927.

Greenstein, J. L.: 1960, Stars and Stellar Systems 6 (ed. by J. L. Greenstein), University of Chicago Press, Chicago, p. 676.

Greenstein, J. L.: 1969, Astrophys. J. 158, 281.

Griem, H.: 1968, Astrophys. J. 154, 1111.

Klemola, A. R.: 1961, Astrophys. J. 134, 130.

Mathews, T. A. and Sandage, A.: 1963, Astrophys. J. 138, 30.

Somerville, W. B.: 1965, Astrophys. J. 141, 811.

Strittmatter, P. A. and Wickramasinghe, D. T.: 1970, Monthly Notices Roy. Astron. Soc., in press (paper III).

Terashita, Y. and Matsushima, S.: 1969, Astrophys. J. 156, 183.

van Horn, H. M.: 1970, Astrophys. J. Letters 160, 53.

Vardya, M. S.: 1961, Astrophys. J. 133, 107.

Wickramasinghe, D. T. and Strittmatter, P. A.: 1969, Astron. Astrophys. 2, 242.

Wickramasinghe, D. T. and Strittmatter, P. A.: 1970, Monthly Notices Roy. Astron. Soc., 150, 435 (paper II). 